\title{
The Work of Secondness as Habit in the Development of Early Schemes
}

\begin{abstract}
Donna E. West
This inquiry demonstrates the pivotal place of attentional phenomena in associating sign, Object, and Interpretant in Secondness. It identifies overt attentional habits (Indexes) and how they are modified/replaced in favor of more covert ones (symbols). The role of Peirce's Object in securing attentional patterns to affairs in Secondness is highlighted. Alterations in use of attentional artifacts determine the kind and degree of reasoning. While use of Indexical signs in Secondness promotes assumptions of co-occurring cause-effect relations, use of symbols in Thirdness facilitates attenuation between cause and effect. As such, agency (human or otherwise) fails to wholly account for assignment of novel Interpretants to ground triads, particularly when the Object does so. Findings illustrate how unexpected eventualities in Secondness call for reconstruction of premises and inductive experimentation, and as such, how recognition of regularities in Secondness re-emerge to renovate patterns of reasoning from diagrammatic to abductive reasoning.
\end{abstract}

Keywords: child development, Dicisign, habit, Index, Peirce, Piaget, Secondness

\section{Introduction}

The single most influential building block of Peirce's semiotic, the element integrating his phenomenology and pragmatist (or pragmaticist) approach, are his categories of Firstness, Secondness, and Thirdness. While they were proposed rather early on in "On a New List of Categories" (1867, EP1, p. 1-10), they, nonetheless, permeate revisions of his approach, later when his pragmatic maxims became primary $(1878,5.402 ; 1903,5.212) .{ }^{1}$ In "On a New List of Categories" (1867, EP1, p. 2), Peirce introduces the seeds of Secondness. He refers to Secondness as a "presence" which he describes as a "substance," as opposed to "being." Here substance consists in objects without "connotation" - entities focused on via attention, but not rising to the level of classifiable objects.

Although Peirce determines Firstness to be the simplest of the categories, given its self-based, autonomous, and non-verifiable nature (1903, EP2, p. 150), he considers Secondness to be the most primary. Its primacy resides in its indispensability to his pragmatist platform - his tychistic conviction that foundational to any advance in logic is its purpose as played out in the regular course of lived experience. "...Secondness is the easiest to comprehend, being the element that the rough-and-tumble of this world renders most prominent" (1903, EP2, p. 268).

Whereas Firstness pertains primarily to rather self-contained internal resurgences of affect and possibility which defy the need for verification or reliability, and while Thirdness (though subject to these considerations) is simply not subject to direct interaction, Secondness particularly lends itself to the core of pragmaticism. As such, manipulation of tangible objects in the physical world enlivens children's experiences via a process of updating raw impressions/percepts. Secondness has far-reaching applications - to experiences of other species and even to natural operations in the physical world, because all are integral to Peirce's master plan, to unify components of the continua. The latter

\footnotetext{
1 "Consider what effects, that might conceivably have practical bearings, we conceive the object of our conception to have. Then, our conception of these effects is the whole of our conception of the object" $(1878,5.402)$. All references to Peirce's unpublished manuscripts follow the convention of the Peirce Edition Project, using the numeral " 0 " as a placeholder when necessary.
} 
illustration of Secondness pertains to principles in science which despite their effect on all constituents of the continua, ${ }^{2}$ do not apply mechanistically (absolutely); rather their occurrence is consonant with what Peirce refers to as habit - patterns of regularity incorporating chance (c. 1890, 1.390). In fact, habit supplies the template to track the most primary of children's early operations with potential objects in Secondness, and is clearly instrumental in describing how children construct object/person concepts initially via sensorimotor schemes.

Accordingly, in Section 2, the fundamental nature of Peirce's category of Secondness is demonstrated, with definitions and relevant illustrations of its place in Peirce's phenomenology and epistemology. In Section 3, Secondness is made relevant to another of Peirce's primary concepts, namely, habit. In Section 4, the discussion centers around how habit emerges in children's sensorimotor period, and how Secondness fosters the development of spatiotemporal competencies during the first year. In Section 5 it is demonstrated how the semiosis of index, as a particular sign, facilitates children's social competencies in the second year and beyond. Secondness provides a necessary forum for indexical signs to unfold and ultimately supplies the raw material upon which children react to external phenomena, and to organize such via index into perspectival templates perceiving what others perceive in expanding multi-modal frameworks.

\section{Foundation: Peirce's concept of Secondness}

Peirce describes Secondness in terms of the following qualities, several of which can be expressed as couplings or attributes intrinsically interconnected as parts of the same whole. When Peirce describes Secondness as "confrontitial" (1903, 7.153), he insinuates the presence of a "brute force (1898, MS 339B, p. 295; 1903, 1.24). Peirce's notion of brute force is equivalent to the unexpected impact of an external object/scene upon the perceptual field of an organism (typically giving rise to conscious notice). Essentially, when an object is salient or when it imposes its presence upon the consciousness, it significantly confronts our mind, brutely. Alternatively, the source of the force can emanate from the organism itself, to brutely impose a change in state upon the near surround, hence effectuating new schemes out of slices of Secondness. "Struggle" (1903: EP 2: 150), and "effort" with "resistance" (1903, 1.24; 1913, L477) characterize just such Secondness-based manipulations. As such, struggle and the "confrontitial", incorporate the sudden and even startling physical experience so characteristic of Secondness. Struggle, effort and resistance materialize upon exertion in the physical surround, especially prominent in the child's early investigative endeavors.

Peirce's further characterization of Secondness as "compulsive" experience (1903, EP2, 268; 1903, 7.674) demonstrates the import of the combinatorial effect of sudden appearances (objects, scenes) confronting the attention, and the immediacy of human reactions to such attentional impositions. This attribute of Secondness integrates his notion of the confrontitial with the issue of the immediate reaction of living organisms. Compulsive experiences can include affirmative or negative responses --either resistance to or acceptance of a state of affairs. Secondness as "hard fact" (1903, EP2, 268), mentioned in the same entry as compulsive experiences consolidate percepts into revisable hypotheses about states of affairs, converting sensory impressions into perceptual judgments/propositions. The brute

\footnotetext{
${ }^{2}$ The neglect of recognizing the influence of habit in the natural and mental worlds is responsible for an overall failure to recognize the import of the continua, as discussed by Pfeifer (in press) - particularly relevant to the establishment of habit as Interpretants in daily events (in lived experience, as well as in the operation of objective, scientific principles). For further discussion of Habit as pertaining to issues of higher thought (West 2014 , in press)
} 
appearance first forces itself upon the attention; only then can propositions as hard facts materialize, grounding scheme formation. An event/experience must first invade the consciousness; only afterward can it be realized as a hard fact. By "hard fact" Peirce intimates that the event is reliable and verifiable, such that it bears some certainty of occurrence/stability, despite perceptual or contextual variants. Although Peirce considers any fact to be a Second - when he refers to the actuality of an event $(1903,1.24)$, he favors those which are confrontitial or compulsive. This is so given their greater likelihood to be noticed and incorporated into hard-fact propositions. Accordingly, Secondness has the potency to capture the attention of sign users, while assimilating all relevant features within the situational context. It draws observers to the existence of particular objects in the here and now - in the daily commerce of events, hinting as to their functionality therein. As such, Secondness initially frames the process of object individuation (by underscoring foregrounded objects), while stenciling the background. In this way, Secondness serves to unite spatial and temporal elements within the physical world by accentuating (via indexical signs) relational regularities particularly incumbent to individual objects which ordinarily self-propel. As such, in Peirce's world of existence in Secondness objects are not passive, inert substances, but active initiators with brute force capacity. They single themselves out, while cementing themselves spatially and temporally to the time and place of the event. In short, Secondness provides the vehicle to be influenced by objects, to operate on them, and to construct event templates - writing on experience the actions and states which the physical world affords.

This interaction with physical entities requires the energy of effort and resistance so characteristic of Secondness. For Peirce, effort and resistance are inextricably linked: "It is inconceivable that there should be any effort without resistance, or any resistance without a contrary effort. This double-sided consciousness is Secondness" (1903, EP2, p. 268). In other words, every action (whether conscious or unconscious) expresses resistance, in that by its very nature effort surfaces as reaction/resistance to something and, at the same time creates novel conditions for subsequent responses. While an impetus (typically a feeling in Firstness) precedes effort (the energy central to a response) the energy itself to coordinate sensorimotor action in Secondness has a more far-reaching influence. Once effort/energy is expressed as sensorimotor coordinated behavior, it induces additional possibilities for effort and resistance in novel schemes (Piaget, 1977/2001, p. 304 n1). As such, efforts in Secondness are not mere static facts/conditions/behaviors, but constitute dynamic, innovative propositions to create novel schemes with their own resistances. Peirce's concept of resistance is hardly limited to conduct in the human sense of acting/reacting. Effort and resistance transcend human agency in self-propelled movement toward goals; they encompass principles involving animates and inanimates in the natural world which materialize as habit (patterns/regularities), constraining/short circuiting/promoting other forces.

Later in his writings, Peirce again connects Secondness with effort in his 1913 letter to F.A. Woods. In fact, effort in Secondness in this passage is embodied as a personal state of being in which effort is given free reign, extending the trope of struggle to the point of hyperbole:

My notion of the second ingredient [Consciousness, Secondness] was gained when I decided that the best means to that end would be to note the state of consciousness when all my muscles were slowly contracting so very much and under such tension as almost to abolish all sensation.... There were two strongly marked states of consciousness. The first was before I lifted [weights equaling one thousand pounds] and was "gathering my forces." It seemed to me that I was subconsciously signaling to dozens of muscles to be ready to respond, all at the same instant, to a signal that would presently be sent. Then I would lift the weight quite slowly and I think about $3 \frac{1}{2}$ or 4 inches. Of course there was a vigorous volition, the consciousness appearing to me exactly like that of applying my mind to anything. 
I described it to myself in bringing an object to distinct focus of conscious while the surrounding unfocused background consisted of self-consciousness. There was a distinct consciousness of oppositions between what consciousness was centered on, and the surrounding vague ego... (1913, L477, p. 23-28).

It is obvious that Peirce, himself, marshals his own, internal effort and resistance against a physical force - to achieve a feat, perceived to be impossible employing ordinary or even superior physical and mental strength. Peirce's purpose in this entry is to accentuate the importance of struggle often necessary when establishing and modifying accomplishments in fields of Secondness. In fact, absent the application of such concerted resistance against supreme physical forces, the potency of Secondness would be poorly understood and virtually unrecognized.

The previous entry indicates that Secondness (articulated in his last description of it) transcends the human condition - since states of affairs were achieved, despite their improbability/impossibility by human agency alone. Peirce's primary claim here is that the most defining attribute of Secondness - that which cuts across all genres of use - is its presence as a state of affairs. Rationale derives from the fact that these states of affairs give rise to facts, and those facts have some objective character; they consist in implicit explanations relating states of affairs to one another (however improbable) - experienced in the personal, social and natural realms, e.g., rocks eroding when proceeding along a trajectory, weights precluding motility). This emphasis on facts and states of affairs demonstrates that Secondness largely encompasses external phenomena. Accordingly, Peirce characterizes Secondness as absent reason $(1903,1.24)$, demonstrating that Secondness is, in large part, mere existence. But, he makes plain that human reaction to states of affairs creates new Secondnesses when he characterizes human effort and resistance as forces to be reckoned with - to modify states of affairs, e.g., anchoring "your shoulder against a door and trying to force it open against an unseen, silent and unknown resistance" $(1903,1.24)$. He emphasizes here that human involvement in the stream of Secondness is anything but passive; rather, expectations for the human role entail a definite call to apprehend and take full advantage of chance, commanding change in states of affairs. Peirce, in fact, depicts this change rather dramatically - as concerted energy against precluded access to Secondnesses in the struggle to push open a door. What precludes the agent from access is a very real, but imperceptible force: an invisible, inaudible, and incognizable barrier.

Although Peirce's explanation of struggle in Secondness illustrates person-to-object exchange, such effort and resistance paradigms extend likewise to person-to-person interactions, as in the removal of a sought-after object from another's grasp or perpetual engagement in an undesirable behavior. The partner responsible for resistance need not be an artifact in the physical world, but may be a social one. Whether animate or otherwise, the partner appears to take on adversarial proportions - often exercising supreme effort to resist a prevailing condition. Schemes requiring resistance are not limited to real-world contexts; they may surface in imaginative and play scenarios. Struggle and resistance can be implemented in conventional or in less conventional contexts, especially when social roles are violated.

\section{Habit paradigms in early fields of Secondness}

Although Peirce's concept of habit is ordinarily associated with Thirdness (as regularity, law, etc.), its effects upon early experience in Secondness is unquestionable. Recognizing the regularity of conditions associated with particular events has its origin in the natural world, as well as in internalized realities in the inner world for Peirce. Two sources/forums (internal as affect/apperception and external entities in the physical surround) exist within which habit 
emerges, underscoring its application to genres of Secondness and relevance to the development of sensorimotor competencies.

Capitalizing on the universal nature of habit, Peirce appears to provide for the existence of five major characterizations: regularity or continuity, coordinating mind and matter, physical/cognitive readiness, tendency or disposition, and habituescence. ${ }^{3}$ This inquiry will concern itself with the initial three characterizations, and especially the second, since they closely lend themselves to the establishment and revision of early sensorimotor experience in Secondness. Regularity within the continuum (recognizing the context as representational of the firmament of the universe at large) obviously has its foundation in Secondness. Such regularity surfaces as early dispositions to notice particular patterns which naturally actualize in experience. It often materializes as the probability to notice sequences of entities situated as distinct but aggregate digits, namely, numerosity (Bloom, 2001, p. 166167). Peirce likewise describes the regularity of habit in Secondness as a "tendency to act" (1898, NEM IV, p. 142). This habit paves the way for establishing sensorimotor schemes (Piaget, 1975/1985, p. 69), which is Secondness at its very core - effort to manipulate physical entities in certain ways to ascertain the greatest success.

The second characteristic of habit (coordinating mind and matter) illustrates how epistemology and ontology are naturally integrated. Even from the outset, infants develop schemes (actions, prehension) in connection with physical objects and their locations (matter), associating actions with objects and places. Coordinating behavior and entities/locations obviates the function of various and sundry entities, piecing together an indexical and iconic diagram of relations in Secondness. This process illustrates how habit lends itself to the creation of novel schemes (guiding iconic memories of objects and locations). Habit serves as the primary coalescing factor uniting mind and matter, ultimately insinuating itself into the world of conscious reflection. In short, coordinating mind and matter via habit demonstrates Peirce's envisionment of the continuum as the communion of self with the physical world. What humans learn from the physical world and from discovery within it is how to formulate increasingly more plausible percepts and perceptual judgments (hypotheses explaining regularities of matter). These increasingly more plausible percepts/perceptual judgments about external phenomena guide children's revisions of workable propositions. ${ }^{4}$ Unquestionably habit is founded upon associations within the continuum emphasizing relations within the inner and the outer world - first associations/relations between the organism and objects in Secondness; later those manufactured in the thought world become increasingly relevant to habit in its most graduated and creative form, habituescence.

Peirce asserts these distinct yet interdependent etiologies of habit in his c. 1897 claim that there exist "ideas of feeling," as well as "acts of reaction:" "There are three categories of being; ideas of feelings, acts of reaction, and habits. Habits are either habits about ideas of

\footnotetext{
${ }^{3}$ Habituescence is a term of Peirce's own coinage - “[The] Third mode of consciousness may be briefly denominated 'The consciousness of taking a habit,' or in one word, 'habituescence.' This, of course, will not in the least imply that we are conscious of the formation of every acquired habit" (c.1913, MS 930, p. 31-32).

${ }^{4} 6.145$ (The Monist, 1892) "Now the mind acts in a way similar to this [hypothetic inference], every time we acquire a power of coordinating reactions in a peculiar way, as in performing any act requiring skill. Thus, most persons have a difficulty in moving the two hands simultaneously and in opposite directions through two parallel circles nearly in the medial plane of the body. To learn to do this, it is necessary to attend, first, to the different actions in different parts of the motion, when suddenly a general conception of the action springs up and it becomes perfectly easy. We think the motion we are trying to do involves this action, and this, and this. Then the general idea comes which unites all those actions, and thereupon the desire to perform the motion calls up the general ideal. The same mental process is many times employed whenever we are learning to speak a language or are acquiring any sort of skill.”
} 
feelings or habits about acts of reaction... The former is the Inner World, the world of Plato's forms. The other is the Outer World, or universe of existents" (c. 1897, 4.157). While "ideas of feeling" emanate from resemblance based relations in the inner world (extracted for narrow focus), "acts of reaction" derive from observation of and participation in the outer world (where otherness treads). Ideas of feeling and acts of reaction are united for Peirce, infusing Firstness into Secondness to arrive at early universal meanings packaged in habits in the physical world. What demonstrates the habit-like nature of operations in the physical world is children's interaction with objects within such world, and the constructive processes which underpin sensorimotor schemes. In fact, the types of sensorimotor coordinations (vision to reach) likewise form the impetus for the creation of new habits, which although grounded in Secondness, are impelled by affect in Firstness and law-like regularity (purpose for the scheme) in Thirdness.

\section{Developmental competencies}

Schemes in Secondness materialize from a brute force presence of the object - which supplies the imperative to problem-solve via informed interactions in the world of existence (the world of facts as pure Seconds). Children operate upon discrete objects by ascribing patterns of action to objects - implicitly associating a primary characteristic (motion) with an entity. These ascribed attributions constitute Peircean habits, which in Piagetian terms are updated when experience demonstrates that accommodations are necessary to account for how objects ordinarily behave in fields of Secondness (Piaget, 1937/1954, p. 405-406).

At $0 ; 2.5$, one of the initial Secondness-based regularities which infants discern is that people move from one place to another independently (Hespos and Baillargeon, 2001, p. 145). The regularity emanating from this fact in Secondness is that entities which can selfpropel (namely persons) can traverse an enclosure and can return to the original enclosure. While this fact was once "brute fact" in Peircean terms, after repeated instantiations it becomes "brute reagent" (1898, MS339B, p. 295). ${ }^{5}$ In other words, the child comes to expect this (e.g., it becomes habitual).

Status as reagent surfaces consequent to imbuing the fact-sign with an Interpretant, supplying an awareness of the typical effects of self-propelled ambulation and recognition that such can proceed in reverse. Here, the reagent of self-propulsion is the observation of people leaving and entering via different doors. These additional observations likewise measure where the person has gone, and the likelihood of his/her return; hence they are reagents. Gaze following likewise emerges at 0;2.5 (Scaife and Bruner, 1975, p. 226) looking along the same path as another. This behavior demonstrates some effort to conform a trajectory to another's, and hence to establish a place to locate items within the continuum. It does not yet rise to the level of object-based or social-based conduct. Although Scaife and Bruner demonstrate some primitive form of gaze-following, such is not sustained and does not rise to the level of preferential looking. It is not until $0 ; 10$ that preferential looking emerges reliably and spontaneously (Corkum and Moore, 1998, p. 32).

At 0;3 infants recognize the legitimacy/permanence of objects within the continuum, in that they begin to follow via gaze the movement of an object (Luo and Baillargeon, 2005, p. 303). This competency reveals that path-following has an Indexical foundation in Secondness, especially in view of its indexical and iconic consolidation in the Dicisign (cf. Stjernfelt, 2014, p. 58-63 for a foundational discussion of Peirce's dicisign). The indexical

\footnotetext{
5 "A person who or thing which is acted upon or reacts to something [is a reagent]; [it is] something which acts as a reactive substance, force, etc" (OED, first usage 1656). Peirce situates reagency as a property of Secondness: "What a Second is depends partly on another, regardless of any third, and thus of any reason. It is brute reagent" (MS 339B, p. 295).
} 
quality of path-following is particularly relevant to individuation of objects in fields of Secondness. Path-following reveals some recognition of the effects of observed objects and the effort imbued in objects within transport scenarios in a sea of other existents. Gazefollowing especially demonstrates an apprehension of indexical properties of Secondness unidirectional source-goal directionality as change in object location. The infant's gaze trajectory reproduces the same path entered into by the object (indexical and iconic-based mimetic signs).

At the same age $(0 ; 3)$ landmarks are utilized to more efficiently locate other objects (Quinn, 1994: 66). At this juncture, landmarks (salient nearby objects or portions of objects) are utilized to recall where the sought-after object was last put to rest (or its typical location). Accordingly, landmarks serve as scaffolds in Secondness which confront the observer and solidify components of the continuum - alerting young observers to the presence and particular location of the target object. In Peircean terminology, landmarks are "confrontitial" in that while they confront the infant with objects and their locations, they likewise relativize them via notice of contextual cues. The confrontitial entails effort and resistance in that the object in the physical world impinges upon the consciousness of the observer, illustrating the influence of objects as brute force agents of habit change in Secondness. Landmarks confront human consciousness, in that they serve as a secondary object to locate another object, enhancing recall of this object's location, and at the same time, acting as a path-finder. Because the location of the landmark is proximate to the sought after object, it constitutes an index, heralding its neighboring counterpart. The effort both of the landmark (to facilitate or point to the sought-after object), and of the observer (to settle upon the near vantage point) places the operation squarely in fields of Secondness.

According to Peirce, "there is nothing that is absolutely confrontitial; although it is quite true that the confrontitial is continually flowing in upon us" (1903, CP 7.153). In other words, while individual objects can be "confrontitial" in that they invade consciousness, they are never without contexts to situate them, hence suggesting their purpose. In short, Secondness supplies children not merely with confrontation in the form of individual objects, but with the flow of the continuum - particularly contextual scaffolds (landmarks) to solidify the object's situatedness. Peirce's focus on the continuum makes prominent the issue of continuity at every turn. As Stjernfelt $(2007$, p. 3$)$ articulates: "The concept of continuity is so central and basic to Peirce that it is not too much to say that he built the whole final version of his philosophy around it."

With refinement of eye-hand coordination at 0;4 (Piaget and Inhelder, 1966/1969, p. 10) infants discover which objects are movable, which carry-able, and which are stationary. They likewise discover that objects (although stationary, not self-propelled) can, with force, be moved via human agency. Similarly, according to Leslie $(1984$, p. 31) the realization that non-self-propelled objects can move upon contact with another emerges at the same age. Apprehending this regularity: object movement consequent to contact from another (human/inanimate moving along a decline) illustrates amplification of Peirce's continuum (states of affairs in Secondness); it demonstrates expansion of the Interpretants of causeeffect events (that stationary objects are displaced consequent to contact with another source). Peirce's characterization of Secondness as "brute reagent" is particularly poignant here, in that the notion of cause- to-move via the addition of an agent is evident. Peirce's use of "brute reagent" puts us in mind of the resultative nature of reagents in Secondness - the effect of the "mix" of the reagent on the immediate context into which it has been introduced. In this vein, Peirce considered "brute reagent" to be a primary function of Secondness. The reagentive character of Secondness is particularly evident in this contact to move scenario, given the spontaneity/unexpected nature of the effect, together with the contribution of the object exerting force to displace the ordinarily stationary object. In MS 339 (1898: 295) Peirce clearly articulates this reagentive function of Secondness: "What a Second is depends 
partly on another, regardless of any third, and thus of any reason. It is brute reagent.... Secondness is brute reaction."

At 0;5, infants demonstrate still more amplified gaze-following behaviors when they trace (with their eyes) object paths to their conclusion/goal/destination (Baillargeon, 1986, p. 17); and soon thereafter, at 0;6-0;7, two related advancements surface: the existence of socalled mental index files (Leslie, Xu, Tremoulet, and Scholl, 1998, p. 13; Leslie and Káldy 2007 , p. 117) and the concept of blocked path (Baillargeon 1986, p. 17). Index files constitute the earliest object files (representations in memory), which are said to account for locativebased features associated with familiar objects in the immediate physical context. Their early emergence demonstrates that the situatedness of objects is more salient than are classificatory features such as color, shape, and the like (Leslie and Káldy, 2007, p. 116-117). Early salience of object situatedness demonstrates the primacy of object individuation before their properties are noticed and before comparisons of property similarities is operational founded upon more iconic, analogy based representations. In any case, the early emergence of the index file is indicative of the profound and sweeping influence of Secondness in constructing the first holistic representations. It spotlights the where of objects, which marks the inception of their place within states of affairs - rudimentary to recognizing spatial relations within physical contexts. In contrast, object comparisons of color/shape do little to validate objects' presence in the surround.

In short, the emergence of these three competencies (prehension, blocked path, path destination) within a relatively short interval (two months) is hardly an accident as they share pivotal underlying assumptions - namely, that stopping points which afford either access and/or a place of rest exist in space; and places of rest are intrinsically associated with objects which cannot self-propel.

At the same time that files are established in the mind for object location, ${ }^{6}$ infants show surprise when a barrier does not preclude passage of an object. An object whose path is blocked is expected not to reach its destination; and when it does proceed beyond the barrier, infants' increases in looking time is often used to measure surprise. Looking time may not reliably measure surprise, although many investigators have incorporated this measure into their design. This is so because (despite its inherently unreliable nature) looking time is a convenient measure; and few other measures exist at this age to indicate infants' state of knowledge/representations. Bremner, Slater, and Johnson (2015) agree that looking time may not be a reliable measure of the state of infants' knowledge, arguing that, "...longer looking may simply indicate detection of a discontinuity or perturbation in the perceptual flow of events with which infants have become familiar" (p. 11). Bremner, et al. (2015, p. 9-10) make the claim that infants were able to better track an occluded object with prior habituation to an unoccluded path; likewise, the infants $($ at $0 ; 4)$ were able to track objects which were occluded over a greater distance but within a shorter amount of time. The upshot is that, as Bremner, et al. observe, "4-month olds perceive trajectory continuity when the perceptual load is reduced by limiting either the temporal or the spatial gap over which the trajectory must be interpolated" (2015, p. 10). What Bremner and colleagues do not explicitly state, however, is that reduction in perceptual load is a direct consequence of limitations in working memory - the shorter interval of occlusion provides fewer "snapshots" of states of affairs in Secondness to be held in WM. The brevity and consolidated effect of spatial and temporal factors on the object concept (object permanence) lends further evidence that the "brute"

\footnotetext{
${ }^{6}$ Leslie and Káldy demonstrate that at ;0.5 infants' reduced of looking time at objects means that they recognize and expect to see the object which appears in their visual field. This recognition is assumed to reveal that infants have already constructed a mental construct of the object in its context with previously remembered attributes, such as location, shape, color, etc.
} 
nature of Secondness is a primary factor underlying the formation of early representations. The short duration accomplished by speed of movement from occluded to unoccluded objects, together with the limited size of (un)occluded objects facilitates notice that upon emergence, the object is the same. As reliance on memory is diminished, reliance on physical instantiation is obviated.

These habits of mind/dispositions materialize consequent to reaction to "hard" facts: "...We talk of hard fact. That hardness, that compulsiveness of experience, is Secondness" (1903, EP2, p. 268). Nonetheless, this hard fact that Secondness must have as its foundation - habits of mind which account for the relatedness of objects - is founded upon perceptual judgments of the interaction of elements within the continuum. This expectation of location and mobility toward a goal illustrates that without these dispositions of mind, elementary facts/intuitions interpreting objective phenomena would be hard pressed to guide early epistemological endeavors. In short, these basic illustrations of physical and mental habit underscore the critical function of developmental milestones in ontogeny - they are pregnant not merely with sparks of Peirce's continuum, but with the allied assertion that all events are teleological (carry purpose).

\section{Relevance of social genres}

The effects of Secondness do not stop with object interaction/observation. Components of Secondness continue to "flow in upon us" (CP 7.153) in our participation in events, as agent, receiver, or instrument (used by another). In fact, effects of Secondness are exemplified in the effort, resistance, and struggle permeating our associations with others and the consequent meanings which embody pragmatic models of epistemology and ontology. The semiosis of dicisigns (signs embodying Indexes and Icons, e.g., targeted reach, in approximating the shape of the hand to the object to be handled) appears to constitute the catalyst toward apprehension of the purpose of joint endeavors. Dicisigns serve to secure another's attention, focusing and refocusing their attention on individuated objects within the continuum.

The emergence of pointing for self (to direct the child's own attention to an object and to resemble the finger of that particular person) at 0;9 (Bates, 1976, p. 61) constitutes another dicisign (apart from or in concert with gaze), scaffolding integration of individual components of Peirce's continuum. It is a pre-social behavior, leveraging self-notice of individual entities in the here and now of the context. As such, objects are individuated within their "universe of existents" $(1903,1.24)$ : "the actuality of an event consists in ... its happening then and there ... The specifications then and there involve all its relations to other existents. The actuality of the event lies in its relations to the universe of existents. ... Actuality is something brute" $(1903,1.24)$. Intersubjective arm extension (reciprocal giving/receiving exchanges) at 0;10 (Carpenter, Nagell and Tomasello, 1998, p. 681) shepherds attentional devices into social arenas - making relevant individuation to event partners and thus validating the salience of brute reagents in Secondness. Here infants commandeer cooperation in the operation of objects' force on their consciousness. At this juncture in development, both cooperative and reciprocal effort in Secondness is exemplified - initiating the back-and-forth effort and resistance so characteristic of Secondness. Peirce illustrates this with conviction even as late as 1913 when he describes lifting (as a young man) 1,000 pounds using concentrated volition, effort, and resistance (L477, quoted at length in the foundation section). The regularity of implementing such an intensive concentration of volition/effort against the state of an object (lifting a heavy object) - to produce a state/condition which appears to be impossible - is the hallmark of many developmental attainments; and the resistance measured by physical and social reciprocal interchanges is integral to advancing semiosis. Intersubjective arm extension in giving and receiving scenarios constitutes but one resistance-based precursor to role-switching/perspective-taking. 
The attainment of bi-directional gaze (two partners mutually looking at each other) at 1;0 (Saylor, 2004, p. 608) and joint gaze toward an object at 1;2 (Tomasello, Carpenter, and Liszkowski, 2007, p. 710) mark the juncture when resistance in Secondness acquires a distinctly social character. As is the case with children's resistance against physical objects/their propelling forces toward determining ontological principles, so too other people impose their distinctive (often opposing) perspectives and will. Children react against or in concert with others' actions, struggling to integrate their own place with that of others in the stream of consciousness. In fact, Peirce indicates that this consciousness is "two-sided" ["effort and resistance" (1903, p 1.24)], in which "struggle" (EP2, p. 150) constitutes the Logical Interpretant.

The regularity of purpose for action at this juncture transcends operation on objects for self alone. Socially motivated resistance consists in changing the focus of another to one's own focus - effecting semiosis from dicisigns in Secondness to more Thirdness-based relations. The Interpretant of the dicisign within unidirectional endeavors conforms to selfbased relations - its indexical character is drawn from simple directions/orientations of representations; and its iconic character derives from the sign's inclination to mimic the source-path-destination trajectory. With the onset of social interplays, the nature of the dicisign incorporates a triangular path: from child to adult to target object (or the reverse), illustrating the effect of agent's attempts to commandeer where and at which object other should look. The reciprocal nature of this process particularly illustrates Peirce's characterization of struggle, since both parties in attention centered scenarios exercise tension upon initiating, moderating and accessing alternative approaches - the hallmark of interactive exchanges. The original agent receives effects of another's attentional preferences - often as brute reagent, e.g., reciprocally rolling a ball between partners. Brutely affecting another may likewise be evidenced when one party attempts to impose his will upon another by means of an implicit or explicit imperative.

The next developmental advance incorporates taking on the behaviors/orientation of another, such that the uniqueness of another's perspective is recreated consequent to envisioning one's self in another's place/orientation. Essentially, another's role is identified and assumed by someone who has not been previously defined by such conduct. Although controversy prevails as to the exact age when pretend and representational play behaviors emerge, there is agreement among investigators that adopting another's single behavior (reproducing it) surfaces earlier than do representational play scenarios. The earliest instances of the former appear at approximately 1;7 when children anthropomorphize a doll, e.g., feeding it, (McCune, 1995, p. 204). Wolf (1982, p. 319) reports that individual behaviors ascribing unconventional uses to objects, e.g., using a pencil as a hammer, surface at 2;0. More recently, Harris (2000, p. 35) documents that representational play behaviors emerge at 2;0. Soon after exercising elementary role play, children rise to a still higher semiotic awareness - codifying shifting roles via language.

A primary measure and tool for advancing the semiosis of reciprocal and diverse role-play is the emergence of demonstratives and personal pronouns (West, 2013). Demonstrative pronouns typically emerge early on at approximately $1 ; 6$, within the initial ten words (Clark, 2009, p. 94). Afterward, demonstrative use becomes productive (applying to speaker's near/far spatial relations) at 2;8 (West, 2011). Demonstratives transcend their initial individuational use, extending to referents located differentially with respect to the person assuming a particular conversational role - that of speaker, and the Interpretants associated with such uses significantly advance from capricious idiosyncratic attention to any object anywhere, to a spatially defined system in which location and orientational features are primary. The latter features demonstratives not merely as pointers of separate objects in a narrow slice of Secondness, but as facilitators of amplified fields of Secondness - enhancing notice of the functionality of neighboring components. This expanded deictic use affords 
access to an organized but dynamic geometric model of the physical and social surround. It is infused with specific and reliable locative properties whose coordinates fit diverse situational applications. The latter use is associated with Peirce's Logical Interpretant, since it encodes not locational demarcations alone, but orientations of social and conversational event participants in all possible orientational permutations. By 3;0, the locational and social roles expressed by personal pronouns such as $I$ and you become productive (West, 1986). At this age, $I$ and you are employed syntactically with several different verbs, demonstrating more than formulaic treatment of speaker-listener roles since they are applied reciprocally and refer to a host of potential players.

Secondness continues to impinge upon this attainment - it supplies sustained continuity with distinctive referents and roles while codifying these shifts via language. As such, Interpretants of these linguistic dicisigns obviate children's underlying mental disposition and readiness - defining space not as purely objective or subjective, but as a dynamic system controlled by pragmatic alterations - of players, locations and of orientations. Mastery of this deictic system requires fine-grained integration of several key sign relations: uniting Index with Icon, i.e., adjusting speaker's gaze trajectory and body location and orientation with codified features from the physical environment. In short, the semiosis of self is grounded in an appreciation not merely of otherness via the exercise of effort and resistance, but is derived from appreciation of a speech and narration placeholder, whose referent changes with each use, but whose function remains stable.

\section{Conclusion}

From the outset, Secondness supplies an essential platform for semiosis to unfold; its influence has far-reaching developmental consequences. Secondness ultimately supplies parameters/limits to embody dynamic forums to exercise participant profiles. It highlights the necessity for internalization of grounded experience, providing ultimate spatial and perspectival templates to anticipate others' attentional forums. Secondness allows the subject to enter into the behavioral and mental habits of others - seeing what they see and thinking what they think, imparting modal perspective. Finally, the recursive character of Secondness demonstrates how the same stimuli engenders different effects at increasingly more advanced developmental levels; as Peirce describes the process, stimuli: "continually flow in upon us," such that Secondness suggests different purposes for relations among participants at different levels of thought development. Early on, Secondness forces attention to objective reality within the physical world, afterward reasserting itself on the social plane and suggesting novel meanings and purposes for attentional devices.

\section{Acknowledgements}

I extend many thanks to the two anonymous reviewers whose helpful suggestions enriched the quality of this manuscript. Likewise, I express gratitude to my colleagues at the Semiotic Society of America conference who commented on my presentation of issues expanded upon in this manuscript. 
Author address

Donna E. West

Department of Modern Languages

227-B Old Main

State University of New York at Cortland

PO Box 2000

Cortland, NY 13045 USA

westsimon@twcny.rr.com

About the author(s)

Donna E. West is Professor of Linguistics and Psycholinguistics at the State University of New York at Cortland (USA). For more than twenty-five years she has been presenting and publishing in the field of Semiotic studies using Peirce's sign system, and has likewise drawn upon semiotic properties in the works of Karl Bühler and Jean Piaget. She is the first investigator to apply a Psycholinguistic developmental perspective to Peirce's ten-fold division of signs. Her 2013 book investigates the role of Index in the acquisition of demonstratives and personal pronouns. She is currently principal editor of an anthology - the first interdisciplinary treatment of Peirce's notion of habit.

\section{References}

Baillargeon, R. (1986). Representing the existence and the location of hidden objects: Object permanence in 6- and 8-month-old infants. Cognition, 23, 21-41.

Bates, E. (1976). Language and context: The acquisition of pragmatics. New York: Academic Press.

Bloom, P. (2001). Roots of word learning. In M. Bowerman and S. C. Levinson (Eds.), Language Acquisition and Conceptual Development, (pp. 159-184). Cambridge: Cambridge University Press.

Bremner, J. G., Slater, A. M. and Johnson, S. P. (2015). Perception of Object Persistence: The Origins of Object Perception in Infancy. Child Development Perspectives, 9(1), 713.

Carpenter, M., Nagell, K., and Tomasello, M. (1998). Social cognition, joint attention, and communicative competence from 9 to 15 months of age. Monographs of the Society for Research in Child Development, Serial 255, 63(4).

Clark, E.V. (2009). First language acquisition (2nd ed.). New York: Cambridge University Press.

Corkum, V., and Moore, C. (1998). The origins of joint visual attention in infants. Developmental Psychology, 34(1), 28-38.

Harris, Paul. (2000). The Work of the Imagination. Oxford: Blackwell Publishers.

Hespos, S. and Baillargeon, R. (2001). Infants' knowledge about occlusion and containment events: A surprising discrepancy. Psychological Science, 12 (2), 141-147.

Leslie, A. (1984). Infant perception of a manual pick-up event. British Journal of Developmental Psychology, 2, 19-32.

Leslie, A. and Káldy, Z. (2007). Things to remember: Limits, codes, and the development of object working memory in the first year. In L. Oakes and P. J. Bauer (Eds.), Short- and Long Term Memory in Infancy and Early Childhood, (pp. 103-125). Oxford: Oxford University Press.

Leslie, A. M., Xu, F., Tremoulet, P., and Scholl, B. (1998). Indexing and the object concept: Developing "what" and "where" systems. Trends in Cognitive Sciences, 2, 10-18. 
Luo, Y. and Baillargeon, R. (2005). When the ordinary seems unexpected: Evidence for incremental physical knowledge in young infants. Cognition, 95, 297-328.

McCune, L. (1995). A normative study of representational play in the transition to language. Developmental Psychology, 31 (2), 198-206.

Peirce, C. S. (i. 1866 - 1913). The Collected Papers of Charles Sanders Peirce, Vols. I - VI eds. C. Hartshorne and P. Weiss (Cambridge, Massachusetts: Harvard University Press 1931 - 1935), Vols. VII - VIII ed. A. Burks (Same publisher, 1958).

Peirce, C. S. (i. 1866 - 1913). The Essential Peirce: Selected Philosophical Writings. Vol. 1, N. Houser and C. Kloesel (Eds.); Vol. 2, Peirce Edition Project, (eds.). Bloomington: University of Indiana Press, 1992-1998.

Peirce, C. S. (i. 1866 - 1913). The New Elements of Mathematics, Vol IV ed. C. Eisele. The Hague: Mouton Press, 1976.

Peirce, C. S. (i. 1866 - 1913). Unpublished manuscripts are dated according to the Annotated Catalogue of the Papers of Charles S. Peirce, R. Robin (ed.). Amherst: University of Massachusetts Press, 1967.

Pfeifer, D. (in press). Charles Peirce, signs, and meaning. In J. Pelkey (ed.), The SSA Annual: Semiotics 2015.

Piaget, J. (1937/1954). The Construction of Reality in the Child. M. Cook (Trans.). New York: Basic Books.

Piaget, J. (1975/1985). The Equilibration of Cognitive Structures. Trans. T. Brown and K. J. Thampy. Chicago: University of Chicago Press.

Piaget, J. (1977/2001). Studies in Reflecting Abstractions. Trans. R.L. Campbell. London: Routledge.

Piaget, J. and Inhelder, B. (1966/1969). The Psychology of the Child. H. Weaver (Trans.). New York: Basic Books.

Quinn, P. C. (1994). The categorization of above and below spatial relations by young infants. Child Development, 65, 58-69.

Saylor, M. M. (2004). Twelve- and 16-month-old infants recognize properties of mentioned absent things. Developmental Science, 7(5), 599-611.

Scaife, M. and Bruner, J. (1975). The capacity for joint visual attention in the infant. Nature, $253,265-266$.

Stjernfelt, F. (2007). Diagrammatology: An Investigation on the Borderlines of Phenomenology, Ontology, and Semiotics. Heidelberg: Springer-Verlag.

Stjernfelt, F. (2014). Natural Propositions: The Actuality of Peirce's Doctrine of Dicisigns. Boston: Docent Press.

Tomasello, M., Carpenter, M. and Liszkowski, U. (2007). A new look at infant pointing. Child Development, 78(3), 705-722.

West, D. (1986). The Acquisition of Person and Space Deictics: A Comparison Between Blind and Sighted Children. Unpublished Doctoral Dissertation, Cornell University.

West, D. (2011). Deixis as a symbolic phenomenon. Linguistik Online, 50(6), 89-100.

West, D. (2013). Deictic Imaginings: Semiosis at Work and at Play. Heidelberg: SpringerVerlag.

West, D. (2014). From habit to habituescence: Peirce's continuum of ideas. In J. Pelkey (ed.), The SSA Annual: Semiotics 2013. Toronto: Legas Press, 117-126.

West, D. (in press). Dialogue as habit-taking in Peirce's continuum: The call to absolute chance. Dialogue (Canadian Review of Philosophy).

Wolf, D.P. (1982). Understanding others: A longitudinal case study of the concept of independent agency. In G. E. Forman (ed.), Action and thought: From Sensorimotor Schemes to Symbolic Operations (pp. 297-327). New York: Academic Press. 